\title{
Cáncer colorrectal durante la pandemia COVID-19, recomendaciones de la Asociación Colombiana de Coloproctología
}

\section{Recommendations of the Colombian Association of Coloproctology for Management of Colorectal Cancer during the COVID-19 Pandemic}

Jorge Mario Castro-Beltrán, MD, ${ }^{\star}{ }^{\star}$ Juan Carlos Reyes-Meneses, MD, ${ }^{2}$ Carlos Edgar Figueroa-Avendaño, MD, ${ }^{3}$ Luis Jorge Lombana-Amaya, MD, ${ }^{4}$ Carlos E. Martínez-Jaramillo, MD, ${ }^{5}$ Eduardo Londoño-Schimmer, MD, ${ }^{6}$ Nairo Javier Senejoa-Núñez, MD, ${ }^{7}$ David Ricardo Baquero-Zamarra, MD, ${ }^{8}$ Javier Alejandro Carrera-Siachoque, MD. ${ }^{9}$

1. Hospital Federico Lleras Acosta, Clínica Medicádiz, Clínica Clinaltec. Ibagué, Colombia. https://orcid.org/0000-0002-7997-7222

Fundación Abood Shaio, Clínica del Country. Bogotá D. C., Colombia. https://orcid.org/00000002-1758-7149

Hospital Universitario Mayor-Méderi, Universidad del Rosario. Bogotá D. C. Colombia.

${ }^{4}$ Hospital Universitario San Ignacio, Clínica de Marly, Universidad Javeriana. Bogotá D. C., Colombia. https://orcid.org/0000-0002-71652124

Hospital Militar Central, Universidad Militar Nueva Granada, Clínica de Marly. Bogotá D. C Colombia. https://orcid.org/0000-0002-49828955

Jefe de sección de cirugía de colon y recto, Departamento de Cirugía, Hospital Universitario Fundación Santafé de Bogotá Profesor Asociado Universidad de los Andes, Universidad El Bosque. Bogotá D. C., Colombia https://orcid.org/0000-0002-0842-6124 Hospital Militar Central, Universidad Milita Nueva Granada, Bogotá D. C., Colombia. https://orcid.org/0000-0002-9785-8534

${ }^{8}$ Epidemiólogo, Hospital Universitario MayorMéderi. Universidad del Rosario. Bogotá D. C. Colombia. https://orcid.org/0000-0002-26094231

9 Hospital Universitario Fundación Santafé de Bogotá, Los Cobos Medical Center, Centro de Coloproctología y Endoscopia Digestiva. Bogotá D. C., Colombia. https://orcid.org/0000 0001-7040-0518

“Correspondencia: Jorge Mario Castro-Beltrán, MD jorgemariocastro@yahoo.com

Fecha recibido: $\quad 15 / 05 / 20$ Fecha aceptado: $05 / 06 / 20$

\section{Resumen}

La pandemia por el coronavirus 2 del síndrome respiratorio agudo severo (SARS-CoV-2), (coronavirus disease-19 [COVID-19]), ha alterado por completo toda la realidad mundial con repercusiones económicas, sociales y grandes compromisos de los sistemas de salud.

La enfermedad ha afectado a todos los países en los 5 continentes; y en nuestro país, desde el primer caso, se han venido tomando medidas para prepararnos mejor ante esta crisis.

Pese a que se trata de un virus respiratorio, se ha documentado su presencia en diferentes tejidos y órganos de los seres humanos. Aunque la presentación clínica en su mayoría tiene síntomas leves, se sabe que un porcentaje importante tiene manifestaciones graves que pueden llevar a complicaciones graves y la muerte.

El cáncer colorrectal es un tumor prevalente en nuestra población y obliga a tener una mejor preparación para tratarlo durante este período.

Desde la Asociación Colombiana de Coloproctología, basados en los diferentes reportes de la literatura, en las recomendaciones de las diferentes asociaciones internacionales y en nuestra propia experiencia, se realiza una revisión del cáncer colorrectal durante la pandemia de COVID-19 y se comparten algunas recomendaciones para el manejo de los pacientes con esta patología revisando las diferentes opciones de manejo según la presentación de la enfermedad.

\section{Palabras clave}

SARS-CoV-2, COVID-19, cáncer colorrectal, guía de manejo.

\section{Abstract}

The economic and social repercussions and the enormous commitment required of health care systems by the severe acute respiratory syndrome coronavirus 2 (SARS-CoV-2) pandemic (coronavirus disease [COVID-19]) has completely altered world reality. The disease has affected all countries on all five continents. In Colombia, from diagnosis of the very first case, measures have been taken to better prepare ourselves for this crisis.

Although it is a respiratory virus, its presence in various human tissues and organs has been documented. Despite the fact that its clinical presentation is most often in the form of mild symptoms, a significant percentage of those infected have severe manifestations that can lead to serious complications and death.

Colorectal cancer is a prevalent tumor in our population, and this pandemic forces to prepare ourselves better to treat it during this period.

The Colombian Coloproctology Association has reviewed reports in the literature and recommendations of various international associations and on our own experience with colorectal cancer during the COVID-19 pandemic. We present our recommendations for management of patients with this pathology and review management options according to disease presentation.

\section{Keywords}

SARS-CoV-2, COVID-19, Colorectal cancer, management guide. 


\section{INTRODUCCIÓN}

Desde su aparición en diciembre de 2019 en la ciudad China de Wuhan, la infección por el coronavirus 2 del síndrome respiratorio agudo severo (SARS-CoV-2), (coronavirus disease-19 [COVID-19]) ha sido para todas las generaciones vivas la patología con mayor impacto mundial para los sistemas de salud, para la economía mundial y para la geopolítica global $(1,2)$. El 11 de marzo de 2020 fue declarado por la Organización Mundial de la Salud (OMS) como pandemia (3).

El COVID-19 es un virus respiratorio que ha mostrado alta contagiosidad y gran dificultad para la detección temprana, tiene diferentes presentaciones que van desde un $80 \%$ de pacientes asintomáticos hasta un $5 \%$ que puede necesitar cuidado intensivo y ventilación mecánica (4). El tratamiento es sintomático y se basa en garantizar una adecuada oxigenación, puede ir desde soporte mínimo con oxígeno $\left(\mathrm{O}_{2}\right)$, ventilación mecánica y membrana de oxigenación extracorpórea (OMEC); también se ha asociado con falla orgánica multisistémica en los casos más graves (5). Múltiples esquemas de manejo médico se han evaluado para contrarrestar el virus: antirretrovirales, antimaláricos, antiparasitarios, medicamentos biológicos, plasma convaleciente $(2,6)$ y otros que se están utilizando con diferentes resultados, que se irán decantando con la experiencia y los resultados alrededor del mundo.

La rápida propagación del virus ha obligado a todos los países a tomar diferentes medidas para tratar de aliviar la pandemia: el lavado de manos, el distanciamiento físico y el uso de tapabocas, como métodos directos de evitar el contagio de persona a persona y las cuarentenas masivas; $\mathrm{y}$ el cierre de fronteras y el aislamiento de grupos de riesgo como medidas epidemiológicas a gran escala para prevenir el crecimiento descontrolado de los enfermos y la saturación de los servicios sanitarios mundiales (6).

En Colombia, las medidas tomadas por el Gobierno Nacional de forma temprana hicieron que disminuyera la presentación esperada de casos y permitió al sistema de salud aumentar su capacidad, entrenar al personal y mejorar la dotación de elementos de protección personal, preparándonos para la parte más grave de la crisis. Desde la Asociación Colombiana de Coloproctología, se acogieron esas medidas, por lo que se redujo nuestra actividad ambulatoria y electiva en las salas de cirugía y en las unidades de endoscopia.

Como cirujanos coloproctólogos, una de las patologías que más se ven y tratan a diario es el cáncer colorrectal (CCR). Debido al gran impacto mundial y la afectación de todos los sistemas de salud, se han establecido recomendaciones basadas en la seguridad de la atención, en la disponibilidad de recursos y en las necesidades de cada institución; todas ellas con el objetivo de tratar de mantener el mejor manejo posible a todos los pacientes.

Cabe resaltar que no hay expertos en esta enfermedad y la experiencia y desarrollo de la pandemia lleva a realizar consideraciones lógicas basadas en lo aprendido, lo esperado y lo vivido.

\section{¿QUÉ HA PASADO CON EL DIAGNÓSTICO DE CCR?}

Debido a la disminución de la actividad endoscópica programada por la pandemia, los nuevos diagnósticos de CCR han disminuido (7). La mayoría de casos que se han atendido corresponden a pacientes con diagnóstico previo o pacientes hospitalizados por síntomas de sangrado, anemia u obstrucción intestinal que han permitido realizar estudios diagnósticos. El debut con obstrucción intestinal o sangrado representa en este momento un porcentaje mayor al habitual de los pacientes operados de cáncer (Figura 1).

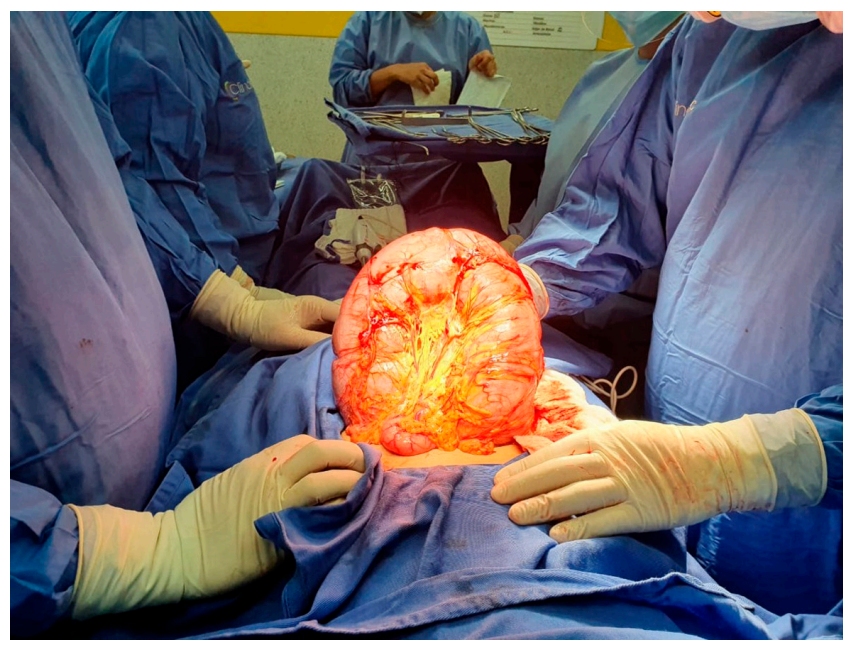

Figura 1. Emergencia quirúrgica, tumor obstructivo del ángulo esplénico del colon.

\section{MANEJO DEL CCR}

\section{Generalidades}

La estadificación del CCR es la piedra angular para definir el plan de tratamiento. Actualmente ha permitido definir prioridades: los tumores tempranos y estadios I no son una emergencia quirúrgica, y dependiendo del momento propio de cada institución y ciudad se pueden contemporizar o llevar directamente al quirófano.

Del mismo modo, los estadios IV solo serán quirúrgicos de inmediato si hay obstrucción o sangrado grave no susceptible de manejo con otros métodos de control; en la enfermedad metastásica el pronóstico está dado por la 
enfermedad sistémica y su control se debe realizar en conjunto con los servicios de oncología (8).

Los estadios II y III de cáncer de colon deben ser llevados a cirugía sin contemporización. La decisión de operar está dada por el momento propio de cada institución, y en esta situación más que nunca es el cirujano quien debe decidir ese momento. Se han establecido fases de acuerdo con la pandemia, dadas por la ocupación del COVID en la institución y la disponibilidad de elementos de protección personal: si la ocupación es menor del $50 \%$ y hay suficientes elementos de protección personal, se pueden programar los pacientes oncológicos; si la ocupación del COVID es mayor, se prefiere diferir o reconsiderar otras opciones y se deja la cirugía solo para emergencias como obstrucción completa, perforación o sangrado masivo (AEC) (9).

La preparación de las instituciones en rutas y áreas de COVID debe ser tenida en cuenta para la seguridad del paciente con patología colorrectal y los protocolos institucionales deben garantizar las áreas seguras en los hospitales. En nuestro medio, las redes de atención de pacientes pueden ser limitadas, pero si existe la oportunidad de dirigir al paciente oncológico a una institución sin pacientes con COVID, puede ser una opción valiosa en algún momento $(7,9)$.

El manejo debe ser individualizado para cada paciente, con base en las diferentes recomendaciones y guías de manejo. Son fundamentales las decisiones de grupo y las juntas quirúrgicas en casos difíciles o cuando haya duda entre varias opciones de manejo, siempre se debe balancear la mejor conducta con la seguridad del paciente y del grupo quirúrgico; los problemas éticos o las decisiones difíciles de tomar deben quedar descritas muy claramente en las historias clínicas.

Actualmente, más que nunca, el consentimiento informado debe ser completo y describir con claridad el riesgo de una cirugía durante la pandemia de COVID-19. Se le deben mencionar al paciente y a su familia los riesgos de cirugías en pacientes asintomáticos o con falsos negativos de pruebas de COVID, el riesgo de infección en la institución de salud y el riesgo de infección posterior al alta. Todos estos riesgos y la discusión con el paciente deben hacer claridad del aumento de complicaciones graves adicionales a los riesgos inherentes a una cirugía colorrectal mayor, y deben quedar consignados en la historia clínica (10).

Se recomienda en esta época de pandemia que los tratamientos los realice el profesional o grupo más entrenado disponible en el medio, y se realicen de acuerdo con la mejor experiencia disponible en cada lugar, basados en la disponibilidad de recursos y de personal.

Se debe tener un plan quirúrgico establecido y alternativas preparadas ante hallazgos inesperados, todas las variaciones deben estar contempladas para garantizar los suministros necesarios con rapidez.
La manera normal de programar los pacientes cambió y en muchos lugares no se cuenta con franjas quirúrgicas formales. La disminución de la cirugía electiva ha hecho necesario reacomodar las agendas de manera tal que se optimice la utilización de quirófanos y se mezclen varias especialidades en un mismo quirófano en el transcurso del día; en todas las salas de operaciones se ha alterado el cumplimiento de los horarios por las precauciones de transporte de pacientes y seguridad de todo el personal.

La implementación de la cirugía con todos los elementos de seguridad personal para pacientes y para el grupo quirúrgico ha aumentado los tiempos de preparación anestésica, de recuperación y de traslado de pacientes; y también se han prolongado los tiempos quirúrgicos, pues las gafas, la respiración con máscara y los cambios en la visibilidad habitual han modificado lo que se venía haciendo por años, sin hablar del cansancio y la fatiga que generan los elementos de protección personal. Todo esto ha afectado el rendimiento habitual (Figuras 2, 3 y 4).

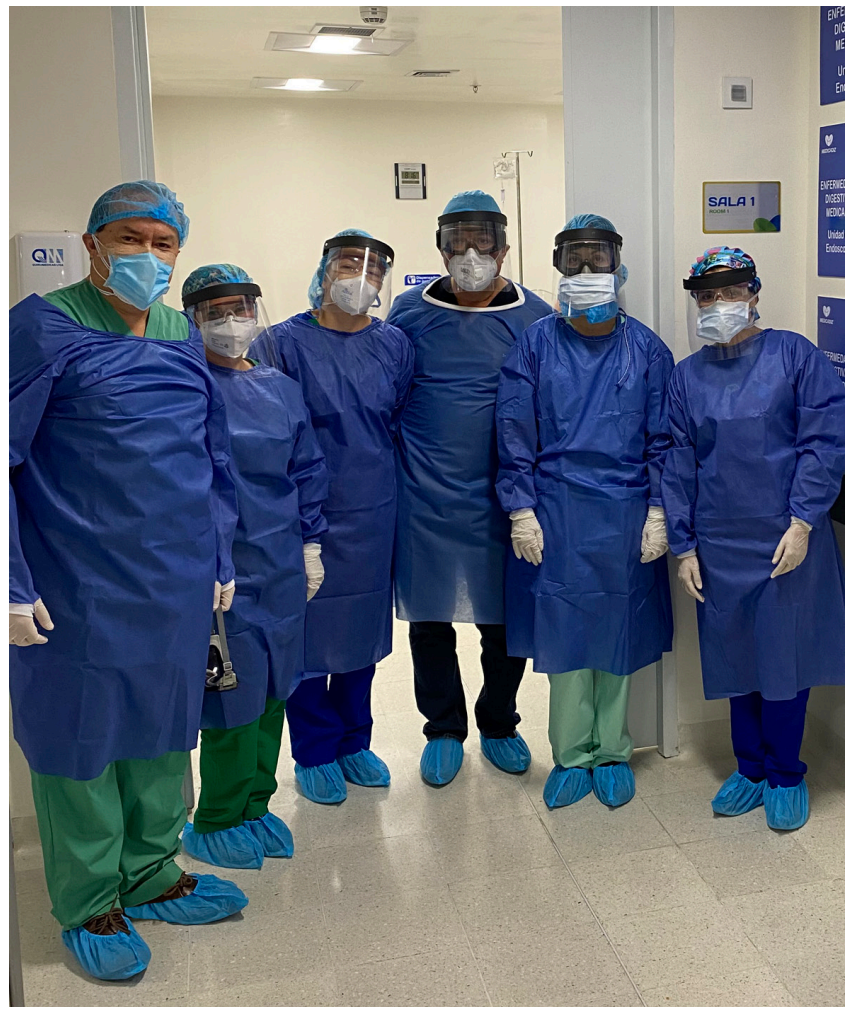

Figura 2. Elementos de protección personal en las salas de endoscopia

A largo plazo se deben determinar las repercusiones médicas, laborales, ocupacionales y emocionales del uso permanente de los elementos de protección personal. Sin lugar a dudas toma más tiempo que antes completar un caso quirúrgico, aspecto fundamental al momento de programar las actividades, pues se debe continuar dismi- 
nuyendo el flujo de personas en los quirófanos y sitios de espera hospitalarios.

Esta situación podrá generar cambios en la en la productividad de las unidades de endoscopia y de los quirófanos. Estas consideraciones se deben tener presupuestadas por los próximos meses y podrían generar nuevas maneras de contratación (Tabla $\mathbf{1}$ ).

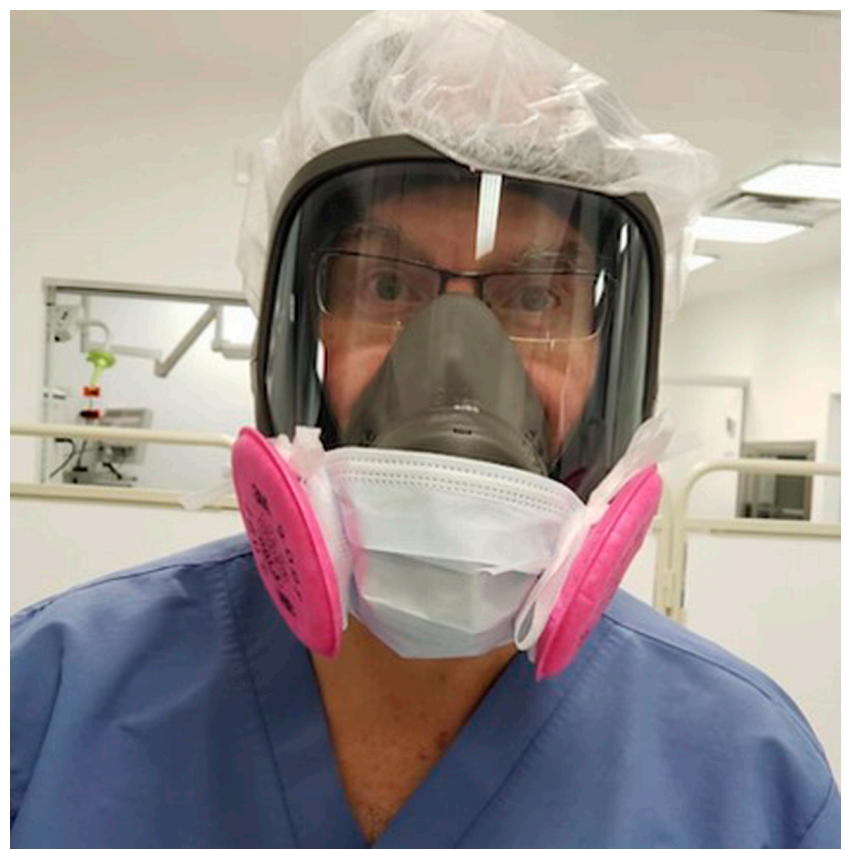

Figura 3. Máscaras elastoméricas para cirugía colorrectal mayor prolongada.

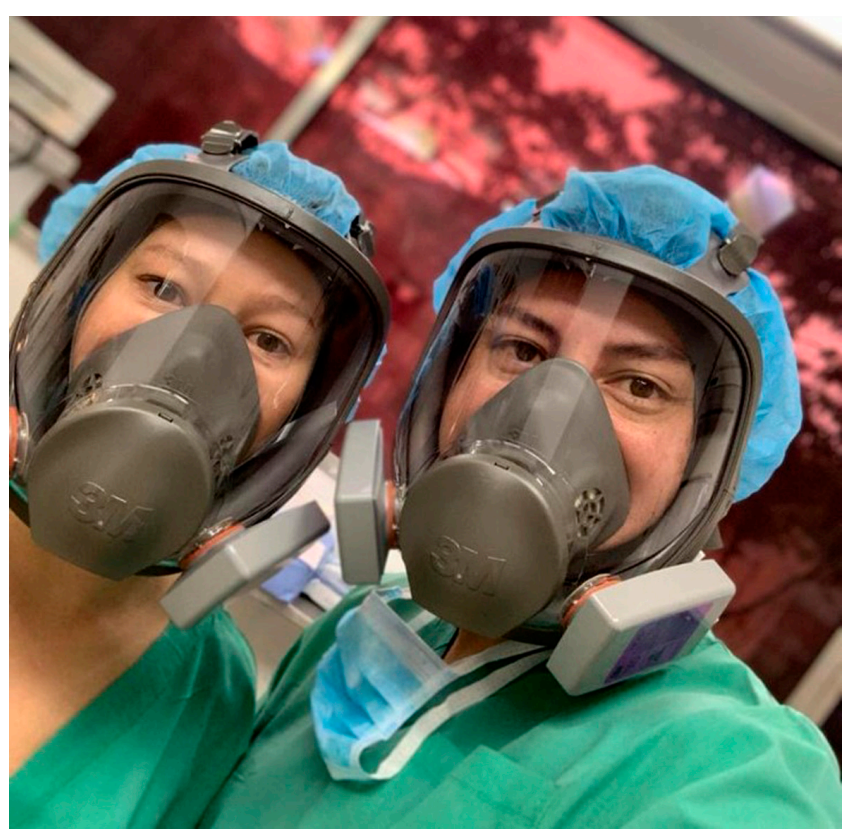

Figura 4. Nueva realidad en elementos de protección personal para el grupo quirúrgico.
Tabla 1. Recomendaciones sobre generalidades en el manejo del CCR

\begin{tabular}{|ll|}
\hline \multicolumn{1}{c|}{ Condición } & \multicolumn{1}{c|}{ Recomendación } \\
\hline CCR estadio I & $\begin{array}{l}\text { Posponer cirugía. Evaluar contexto local y si } \\
\text { este es favorable (baja ocupación UCI): cirugía }\end{array}$ \\
\hline CCR estadio IV & $\begin{array}{l}\text { Únicamente cirugía urgente: perforación, } \\
\text { obstrucción, sangrado grave }\end{array}$ \\
\hline CCR estadios II y III & Manejo quirúrgico. Evaluar el contexto local \\
\hline $\begin{array}{l}\text { Todos los casos de } \\
\text { CCR }\end{array}$ & $\begin{array}{l}\text { Respaldar la conducta en junta de decisiones } \\
\text { medicoquirúrgica }\end{array}$ \\
\hline Todos los pacientes & $\begin{array}{l}\text { Información y consentimiento informado de } \\
\text { COVID }\end{array}$ \\
\hline Personal de salud & $\begin{array}{l}\text { Uso rutinario de elementos de protección } \\
\text { personal }\end{array}$ \\
\hline
\end{tabular}

UCI: unidad de cuidados intensivos.

\section{Resección endoscópica}

El tratamiento del CCR es resectivo en la mayoría de los casos. En lesiones premalignas y tumores tempranos con ciertas características histológicas y morfológicas, se puede intentar una resección endoscópica; sin embargo, en las fases iniciales de la pandemia con la suspensión de las colonoscopias electivas estos pacientes pudieron quedar en espera, por lo que se debe considerar una reevaluación endoscópica y, si se mantiene la condición inicial, intentar la resección endoscópica con las técnicas de mucosectomía o disección endoscópica submucosa, dependiendo de la experiencia del grupo tratante (11).

La colonoscopia se considera productora de aerosoles y la presencia de ácido ribonucleico (ARN) de COVID-19 en las heces hacen que se requieran las máximas precauciones con los elementos de protección personal e idealmente hacer uso de salas de endoscopia con presión negativa (12-14).

En caso de que no sea candidato a resección endoscópica, se debe llevar el paciente a cirugía; cada institución y ciudad evaluará su propia situación para decidir el momento de realizar el procedimiento.

\section{Resección local}

Las lesiones tempranas en cáncer de recto medio o distal pueden ser susceptibles de resección local y los criterios son los mismos de siempre: tumores pequeños, bien diferenciados, con características histológicas favorables y se pueden abordar por técnica abierta por vía transanal. Se requiere máxima protección con los elementos de protección personal por tener exposición directa a la mucosa expuesta con materia fecal y generación de aerosoles por el 
uso del electrobisturí. No hay claridad sobre el sellamiento completo de los dispositivos de cirugía mínimamente invasiva transanal, por lo que la cirugía transanal mínimamente invasiva (TAMIS) es un procedimiento restringido durante la pandemia hasta que no se tenga mayor información sobre su seguridad (Tabla 2) $(9,15)$.

Tabla 2. Recomendaciones sobre la resección endoscópica y la resección transanal

\section{Condición}

Lesiones susceptibles de Reevaluar estas lesiones

resección endoscópica endoscópicamente

antes de la pandemia

Personal de salud

Uso de elementos de protección personal durante los procedimientos endoscópicos y resecciones transanales

TAMIS

Se desaconseja la realización de este procedimiento

\section{Neoadyuvancia y cáncer de recto}

No hay duda de que durante la pandemia, en ausencia de obstrucción, los tumores avanzados de recto se benefician del uso de neoadyuvancia. Se pueden consensuar en juntas multidisciplinarias conductas de quimioterapia de inducción o consolidación, o terapia neoadyuvante total. En algunos centros se ha propuesto realizar esquemas cortos de radioterapia para disminuir el número de sesiones y limitar el riesgo de infección de COVID por desplazamientos o contactos (16).

Los pacientes que terminen la neoadyuvancia y requieran cirugía se pueden operar entre las semanas 6 y 12 siempre que las condiciones de la institución lo permitan; se puede alargar el intervalo hasta la semana 16.

Si se sospecha un tumor T2N0 en el recto medio e inferior, posiblemente sea la oportunidad de ampliar los criterios de neoadyuvancia a este grupo de pacientes como una opción terapéutica, teniendo el sustrato académico de la mayor opción de preservación del órgano y evitarle una cirugía al paciente en casos de respuesta clínica completa $(17,18)$. Es posible que esta experiencia pueda generar nuevas conductas del uso de neoadyuvancia en los tumores del recto superior.

\section{Cáncer de colon}

Para esta entidad, la cirugía es el tratamiento de elección inicial, pero en casos de lesiones grandes con compromiso de órganos adyacentes, donde se vaya a requerir una resección multivisceral mayor, se puede considerar la utilización de quimioterapia neoadyuvante (19).
En los casos de cáncer de colon no obstructivo estadios II y III, según la disponibilidad de camas en cuidados intensivos y el porcentaje de ocupación de las UCI por pacientes con COVID y de camas hospitalarias, se deben llevar los pacientes a cirugía.

Se deben evaluar los predictores de complicaciones y de fístulas en el preoperatorio: la desnutrición, diabetes, uso crónico de esteroides, antiangiogénicos y la condición hemodinámica del paciente, entre otros, deben llevar a ser prudentes en el momento de decidir una anastomosis, por lo que una derivación puede evitar complicaciones graves que aumenten la morbimortalidad. El paciente debe estar siempre informado de estas condiciones y durante la pandemia y sus diferentes fases se debe optar por disminuir los riesgos y hacer más predecibles los postoperatorios, con el fin de evitar reintervenciones y acortar estancias hospitalarias. Probablemente, en este tiempo, más que nunca, se debe balancear la experiencia clínica con la evidencia disponible (20).

\section{PROGRAMACIÓN DE LA CIRUGÍA}

Idealmente, se debe descartar siempre la infección de COVID-19. Las guías internacionales piden realizar pruebas preoperatorias al paciente oncológico colorrectal con 24 a 48 horas de anticipación. No se puede programar ningún paciente sintomático respiratorio, con síndrome febril o contacto cercano de infección de COVID confirmada o sospechosa. Las guías existentes dejan pruebas moleculares y serológicas como alternativas válidas que dan seguridad.

Todo paciente debe tener un cuestionario sobre antecedente de exposición a COVID en su familia o círculo cercano; se debe preguntar sobre viajes y cumplimiento del aislamiento social, con especial énfasis en los riesgos de infecciones asintomáticas.

Se han elaborado métodos predictores de la necesidad de cirugía como la escala Medically-Necessary Time-Sensitive (MeNTS), que evalúa y da puntuación a múltiples variables, y es una ayuda clara sobre la pertinencia de la cirugía (21).

Hasta el momento de esta publicación, en nuestro medio no se ha tenido opción universal de pruebas preoperatorias, por lo que en algunos centros se le ha pedido a los pacientes un aislamiento estricto de 14 días antes de la cirugía y, en ausencia de síntomas, se han operado con la máxima precaución de elementos de protección personal. Nuestros resultados con esta opción empírica han sido satisfactorios sin tener contagios de COVID en el postoperatorio, teniendo en cuenta que estamos en fases iniciales de la pandemia sin llegar a los picos de contagio.

La tomografía de tórax ha sido una opción útil para detectar hallazgos precoces de compromiso viral en el pulmón y, ante la ausencia de pruebas o certeza del aislamiento, es una opción disponible fácilmente (Tabla 3). 
Tabla 3. Recomendaciones sobre la programación de la cirugía

\begin{tabular}{ll}
\multicolumn{1}{c}{ Condición } & \multicolumn{1}{c}{ Recomendación } \\
\hline $\begin{array}{l}\text { Todos los } \\
\text { pacientes }\end{array}$ & $\begin{array}{l}\text { Realización de prueba para la detección del COVID } \\
\text { de forma preoperatoria si está disponible. TAC tórax } \\
\text { es una opción }\end{array}$ \\
$\begin{array}{ll}\text { Programación } \\
\text { de cirugía }\end{array}$ & $\begin{array}{l}\text { No programar sintomáticos respiratorios o con nexo } \\
\text { epidemiológico }\end{array}$ \\
$\begin{array}{ll}\text { Programación } \\
\text { de cirugía }\end{array}$ & $\begin{array}{l}\text { Realización de cuestionario sobre COVID, } \\
\text { aislamiento preventivo 14 días antes de la cirugía }\end{array}$ \\
\hline
\end{tabular}

\section{ELECCIÓN QUIRÚRGICA}

La presencia de ARN viral en el líquido peritoneal y en las heces ha sido confirmada $(22,23)$; sin embargo, su contagiosidad no se ha demostrado, y esto obliga a tomar las máximas precauciones quirúrgicas. No hay certeza de la contagiosidad de los aerosoles producidos durante la cirugía en la cavidad peritoneal y existen pros y contras de las diferentes técnicas y abordajes.

Durante la pandemia y en la fase en la que estamos se recomienda utilizar todos los elementos de protección recomendados por las diferentes agencias que se han pronunciado al respecto, como el Centro de Control de Enfermedades de Estados Unidos (CDC) y el American College of Surgeons $(24,25)$. El uso de tapabocas N95, monogafas, careta o visor, doble guante y bata antifluido es obligatorio para las cirugías y colonoscopias y se debe ser estricto en la manera de colocar y retirar todos los elementos de protección personal $(26,38)$. Se recomienda realizar los procedimientos, tanto colonoscopias como cirugías, en salas con presión negativa (Figura 5) (27).

\section{Cirugía abierta}

Inicialmente se consideró la vía más segura; se escucharon recomendaciones de China, Estados Unidos y Europa, que llamaron a la adopción de esta vía.

Para evitar escapes inadvertidos de aerosoles contaminantes, se disminuyó al máximo la utilización de energía para disección y hemostasia (28); la tijera, el bisturí y las ligaduras volvieron a ser una realidad en los quirófanos. Esto significa un cambio grande del ejercicio, pues sin duda alguna, los beneficios hemostáticos de las diferentes alternativas existentes acortan tiempos y disminuyen sangrados. Sin embargo, ante la ausencia de información, aún hoy se puede decir que es la técnica con menos riesgos teóricos si se limita el uso del electrobisturí y de la energía bipolar o ultrasónica. No obstante, las nuevas generaciones de cirujanos podrían tener menos experiencia con cirugía sin dispositivos de energía, e indudablemente su uso masivo puede tener con- secuencias negativas con respecto a los sangrados y la prolongación del tiempo quirúrgico. También se tiene certeza de mayor dolor en el postoperatorio y mayor estancia hospitalaria, los cuales son puntos importantes a tener en cuenta en época de necesidad de camas en las instituciones.

\section{Cirugía laparoscópica}

El neumoperitoneo con dióxido de carbono $\left(\mathrm{CO}_{2}\right)$ a presiones de $15 \mathrm{~mm} \mathrm{Hg}$, confinado en una cavidad con humo y aerosoles a mayor presión que el ambiente, genera un riesgo alto de escapes inadvertidos de aerosoles potencialmente contaminantes (27), especialmente cuando históricamente los hemos dejado salir libremente a nuestra sala de cirugía de forma rutinaria.

En la Asociación Colombiana de Coloproctología, la primera recomendación en la fase inicial de la pandemia fue evitar la cirugía laparoscópica en pacientes con COVID o altamente sospechosos por estas consideraciones. Repetimos que no se ha comprobado la generación de aerosoles contaminantes en pacientes con COVID, pero resulta lógico pensar en este riesgo y prevenirlo. Como respuesta a este problema, los cirujanos del mundo rápidamente replantearon la seguridad de la cirugía mínimamente invasiva y empezaron a describir la extracción de humo con diferentes métodos artesanales y algunos técnicamente desarrollados para este fin $(29,30)$.

Los extractores de humo, aunque se habían desarrollado desde antes, no encontraron un momento más oportuno para ser redescubiertos y lanzados; solo ahora fuimos conscientes nuevamente de los potenciales daños, ya descritos y pasados por alto, del humo quirúrgico abierto o laparoscópico en la salud del cirujano y su grupo quirúrgico. Se ha demostrado que puede ser equivalente a fumar (31) y se ha comprobado la presencia de virus de hepatitis $\mathrm{B}$ y del virus del papiloma humano (VPH) $(32,33)$. Indudablemente, estos dispositivos serán un requerimiento obligatorio en los quirófanos del mundo luego de la pandemia.

Es importante recordar algunas lecciones aprendidas de los 10 años de experiencia con la quimioterapia intraperitoneal por laparoscopia. Algunos sistemas de insuflación de $\mathrm{CO}_{2}$ pueden reabsorberlo para proteger el aumento de la presión intraabdominal; por lo tanto, se puede recomendar tener un filtro en el circuito de ingreso al paciente, $y$ también el sistema extractor de humo debe tener un filtro para partículas. Tradicionalmente, los filtros HEPA (high efficiency particulate air) atrapan los diferentes tamaños de partículas portadoras de virus, por lo que su implementación debe garantizarse.

Con el control del humo y los aerosoles se soluciona el problema más importante y se vuelven a ver los grandes beneficios de la cirugía mínimamente invasiva. Para este 


\begin{tabular}{|c|c|c|c|c|c|c|c|c|c|c|c|c|}
\hline \multicolumn{13}{|c|}{$\begin{array}{c}\text { ELEMENTOS DE PROTECCIÓN PERSONAL QUE DEBEMOS USAR EN LA ATENCIÓN DE } \\
\text { PACIENTES DURANTE LA PANDEMIA COVID-19 }\end{array}$} \\
\hline \multirow{2}{*}{$\begin{array}{l}\text { Cirugía/otros } \\
\text { procedimientos }\end{array}$} & & & $=$ & E & & & & & & & & \\
\hline & $\begin{array}{c}\text { Higiene de } \\
\text { manos }\end{array}$ & $\begin{array}{c}\text { Gorro } \\
\text { quirưrgico }\end{array}$ & $\begin{array}{l}\text { Respirador } \\
\text { N95 }\end{array}$ & $\begin{array}{l}\text { Mascarilla } \\
\text { quirurgica }\end{array}$ & Monogafas & Careta & $\begin{array}{l}\text { Vestido } \\
\text { quirúgicico o } \\
\text { uniforme }\end{array}$ & $\begin{array}{c}\text { Bata } \\
\text { antifluidos }\end{array}$ & Guantes & $\begin{array}{l}\begin{array}{l}\text { Polainas } \\
\text { o calzado } \\
\text { especial }\end{array} \\
\end{array}$ & $\begin{array}{l}\text { Peto o } \\
\text { delantal }\end{array}$ & $\begin{array}{l}\text { Escafandra } \\
\text { desechable }\end{array}$ \\
\hline $\begin{array}{l}\text { Médicos (cirujanos, ayudantes) } \\
\text { durante procedimientos quirúrgicos } \\
\text { generadores de aerosoles }\end{array}$ & & & & & $\begin{array}{c}\text { Cualquier } \\
\text { elen }\end{array}$ & & & & & & & \\
\hline Médico anestesiólogo & & 1 & & & & & & & & 4 & & \\
\hline $\begin{array}{l}\text { Instrumentador durante } \\
\text { procedimientos quirúrgicos } \\
\text { generadores de aerosoles }\end{array}$ & & & & & $\begin{array}{l}\text { Cualquier } \\
\text { elen }\end{array}$ & & & & & & & \\
\hline $\begin{array}{l}\text { Enfermera, auxiliar de enfermería } \\
\text { durante procedimientos quirúrgicos } \\
\text { generadores de aerosoles }\end{array}$ & & & & & & & & & & & & \\
\hline $\begin{array}{c}\text { Áreas de trabajo de parto, sala de } \\
\text { partos y cesárea }\end{array}$ & & & & & $\begin{array}{r}\text { Cualquier } \\
\text { elen }\end{array}$ & & & & & & & \\
\hline $\begin{array}{c}\text { Todo el personal asistencial en } \\
\text { áreas de circulación de área } \\
\text { quirúrgica }\end{array}$ & & & & & & & & & & & & \\
\hline Servicios generales & & & & & $\begin{array}{c}\text { Cualquier } \\
\text { elen }\end{array}$ & & & & $\begin{array}{l}\text { Uso de guant } \\
\text { de caucho }\end{array}$ & & $\begin{array}{c}\text { Abundantes } \\
\text { liquidos }\end{array}$ & \\
\hline $\begin{array}{l}\text { Administrativos (incluye biomédica, } \\
\text { mantenimiento, auditoría, etc.) }\end{array}$ & & & & & & & & & & & & \\
\hline Procedimientos odontológicos & & & & & $\begin{array}{c}\text { Cualquier } \\
\text { elen }\end{array}$ & & & & & & & \\
\hline $\begin{array}{l}\text { Endoscopias digestivas, de vías } \\
\text { aéreas superiores, de vías aéreas } \\
\text { inferiores }\end{array}$ & & & & & $\begin{array}{c}\text { Cualquier } \\
\text { elen }\end{array}$ & & & & & & & \\
\hline $\begin{array}{l}\text { Procedimientos invasivos con } \\
\text { requerimiento de videofluoroscopia } \\
\text { y protección de elementos } \\
\text { plomados }\end{array}$ & & & & & $\begin{array}{c}\text { Cualquier } \\
\text { elen }\end{array}$ & $\begin{array}{l}\text { elos dos } \\
\text { tos }\end{array}$ & & & & & 3 & \\
\hline
\end{tabular}

Figura 5. Elementos de protección personal (38)

particular, se puede asumir teóricamente el beneficio de tener una barrera natural que confine a la cavidad peritoneal todos los aerosoles potencialmente contaminantes. Con este fin se recomienda bajar la presión habitual de neumoperitoneo de 8 a $12 \mathrm{~mm} \mathrm{Hg}$ y ser estricto en las incisiones pequeñas y en la hermeticidad de los trócares; se deben evitar los reúsos y adquieren valor los trócares con sistemas de sellado y balones inflables para evitar extracciones acci- dentales (34). El sistema de succión quirúrgica normal de la sala debe tener filtros de partículas al final del sistema colector para evitar la salida de aerosoles y micropartículas al aire ambiente de la sala de cirugía.

La extracción de las piezas quirúrgicas obliga a evacuar previamente todo el neumoperitoneo y tener cuidado especial en ese momento, se recomienda por algunos expertos realizar las anastomosis intracorpóreas para limi- 
tar la exposición del lumen intestinal (9). Se deben minimizar las maniobras que permitan el escape no controlado de gas fuera de la cavidad abdominal como el recambio de gasas y de instrumentos, y usar posiciones menos forzadas del paciente.

La cirugía mínimamente invasiva con protocolos de recuperación rápida produce menos dolor en el postoperatorio y disminuye la estancia hospitalaria, gran ventaja en tiempos de pandemia (35). Las técnicas de resección total del mesorrecto por vía transanal (TaTME) y cirugía transanal mínimamente invasiva (TAMIS) no se recomiendan hasta que no se tenga mayor información por su característica de mezclar la producción de aerosoles con la exposición del lumen en una plataforma en la que es más difícil garantizar el sellamiento $(9,27)$.

Se pueden utilizar suturas absorbibles para el cierre de las incisiones de piel y así posponer la consulta de control y retiro de puntos; la telemedicina se convierte en una excelente alternativa para tener al paciente en casa más tiempo (35). Los signos de alarma deben ser reforzados para evitar consultas tardías por temor a acudir al servicio de urgencias en épocas de pandemia (36) y se podría ver al paciente cuando esté el resultado de patología para explicar los hallazgos y definir la conducta a seguir (Tabla 4).

Tabla 4. Recomendaciones para cirugía

\begin{tabular}{ll}
\multicolumn{1}{c}{ Condición } & \multicolumn{1}{c|}{ Recomendación } \\
\hline $\begin{array}{l}\text { Cirugía } \\
\text { laparoscópica }\end{array}$ & $\begin{array}{l}\text { Disminución de la presión del neumoperitoneo. } \\
\text { Extracción cuidadosa del } \mathrm{CO}_{2}\end{array}$ \\
\hline Técnica quirúrgica & $\begin{array}{l}\text { Continuar con la de mayor familiaridad por grupo } \\
\text { quirúrgico }\end{array}$ \\
\hline $\begin{array}{l}\text { Evacuación de } \\
\text { humo }\end{array}$ & $\begin{array}{l}\text { Evaluar la posibilidad de conseguir evacuadores } \\
\text { de humo o crear uno propio (disponible) }\end{array}$ \\
\hline Técnicas TaTME y & Se desaconseja su uso por ahora \\
TAMIS &
\end{tabular}

\section{REINICIO DE ACTIVIDADES Y NORMALIZACIÓN DEL EJERCICIO DURANTE LA PANDEMIA}

Cada lugar tiene su propia realidad. En China se ha propuesto un funcionamiento del $80 \%$ de la capacidad instalada, en Europa se reabren los servicios y se emiten recomendaciones de cómo regresar de forma segura a las actividades asistenciales (9). Todas ellas implican interrogatorios sobre antecedentes, enfermedad actual, descar- tar fiebre y pruebas específicas para COVID. En nuestro medio, las medidas tomadas por el Gobierno Nacional tuvieron unos resultados esperados en el aplanamiento de la curva de contagio y brindaron tiempo al sistema de salud para ampliar su capacidad instalada, adquirir los elementos de protección personal y entrenar al personal sobre la mejor manera de afrontar esta crisis. Sin embargo, todavía se espera un repunte de la enfermedad, por lo que hablar del reinicio de actividades durante el pico puede ser algo controversial y posiblemente se deba hablar de aprender a trabajar durante la pandemia. Dicho lo anterior, se espera la reapertura ordenada de los servicios ambulatorios y algunas actividades quirúrgicas electivas, de modo que se permita evacuar la atención de pacientes en listas de espera sin comprometer los servicios indispensables de las instituciones, y siempre con la evaluación activa de la ocupación y compromiso del COVID en cada ciudad e institución.

El interrogatorio sobre factores de riesgo y presencia tanto de síntomas asociados como de fiebre se convierte en una pieza fundamental obligatoria para poder atender a los pacientes. La utilidad de las pruebas en el paciente asintomático no es $100 \%$ segura, pero su realización mejora la opción de descartar la infección y da tranquilidad al paciente y al grupo tratante cuando se vaya a llevar a cirugía.

El impacto económico para instituciones y médicos ha sido enorme. En nuestro medio se puede prolongar la duración de la enfermedad, por lo que la atención de pacientes durante las diferentes fases de la pandemia lleva a un nuevo ejercicio de la coloproctología con la apertura parcial de la consulta, la actividad endoscópica y la cirugía electiva, manteniendo los cuidados máximos y reorganizando las agendas de manera tal que se continúe trabajando con la mayor calidad y total seguridad.

Todas estas actividades implican un aumento del tiempo y disminución de la productividad que seguramente deben ser evaluados y compensados por los diferentes actores del sistema de seguridad social. Se espera una revisión de los costos directos e indirectos, incluido el reconocimiento del mayor tiempo en la prestación de servicios profesionales que se verán afectados por este nuevo orden mundial.

La experiencia de otros continentes y países ha permitido ver todos los escenarios, tomar las precauciones y hacer las pausas requeridas con el fin de adaptarnos a nuestra nueva realidad. Se reconoce la posibilidad de una segunda ola de la pandemia, por lo que nuestro ejercicio debe estar preparado ante las diferentes variables de la enfermedad mientras se tienen soluciones globales con vacunas y tratamientos efectivos. 


\section{REFERENCIAS}

1. Zhu N, Zhang D, Wang W, Li X, Yang B, Song J, Zhao X, Huang B, Shi W, Lu R, Niu P, Zhan F, Ma X, Wang D, Xu W, Wu G, Gao GF, Tan W; China Novel Coronavirus Investigating and Research Team. A Novel Coronavirus from Patients with Pneumonia in China, 2019. N Engl J Med. 2020;382(8):727-733. https://doi.org/10.1056/NEJMoa2001017

2. Gosain R, Abdou Y, Singh A, Rana N, Puzanov I, Ernstoff MS. COVID-19 and Cancer: a Comprehensive Review. Curr Oncol Rep. 2020 May 8;22(5):53. https://doi.org/10.1007/s11912-020-00934-7

3. Director-General's opening remarks at the media briefing on COVID-19 - 11 March 2020 [internet]. World Health Organization; 2020 [acceso el 3 abril de 2020]. Disponible en: https://www.who.int/dg/speeches/detail/ who-director-general-s-opening-remarks-at-the-mediabriefing-on-covid-19---11-march-2020

4. Adhikari SP, Meng S, Wu YJ, Mao YP, Ye RX, Wang QZ, Sun C, Sylvia S, Rozelle S, Raat H, Zhou H. Epidemiology, causes, clinical manifestation and diagnosis, prevention and control of coronavirus disease (COVID-19) during the early outbreak period: a scoping review. Infect Dis Poverty. 2020;9(1):29. https://doi.org/10.1186/s40249-020-00646-x

5. Zaim S, Chong JH, Sankaranarayanan V, Harky A. COVID-19 and Multiorgan Response. Curr Probl Cardiol. 2020;45(8):100618.

https://doi.org/10.1016/j.cpcardiol.2020.100618

6. Hernández-García I, Giménez-Júlvez T. Assessment of Health Information About COVID-19 Prevention on the Internet: Infodemiological Study. JMIR Public Health Surveill. 2020;6(2):e18717. https://doi.org/10.2196/18717

7. Ghignone F, Mohan HM, Montroni I. Cancer surgery in a time of COVID-19: Many questions, few certainties. Eur J Surg Oncol. 2020:S0748-7983(20)30442-X. https://doi.org/10.1016/j.ejso.2020.04.046

8. Ren X, Chen B, Hong Y, Liu W, Jiang Q, Yang J, Qian $\mathrm{Q}$ Jiang C. The challenges in colorectal cancer management during COVID-19 epidemic. Ann Transl Med. 2020;8(7):498.

https://doi.org/10.21037/atm.2020.03.158

9. Asociación Española de Coloproctología (AECP). Recomendaciones de actuación patología colorrectal de la AECP ante COVID-19 [Internet]. 2020 [acceso el 3 de marzo de 2020]. Disponible en: https://aecp-es.org/index.php/recomendaciones

10. Maíllo C, Ramos JL, Landac I, Gómez-Alonso A, MorenoAzcoita M. Empleo de los documentos de consentimiento informado por los servicios de cirugía españoles. Cir Esp. 2002;71(6):269-275. https://doi.org/10.1016/S0009-739X(02)71980-5
11. Hashiguchi Y, Muro K, Saito Y, Ito Y, Ajioka Y, Hamaguchi T, Hasegawa K, Hotta K, Ishida H, Ishiguro M, Ishihara S, Kanemitsu Y, Kinugasa Y, Murofushi K, Nakajima TE, Oka S, Tanaka T, Taniguchi H, Tsuji A, Uehara K, Ueno H, Yamanaka T, Yamazaki K, Yoshida M, Yoshino T, Itabashi M, Sakamaki K, Sano K, Shimada Y, Tanaka S, Uetake H, Yamaguchi S, Yamaguchi N, Kobayashi H, Matsuda K, Kotake K, Sugihara K; Japanese Society for Cancer of the Colon and Rectum. Japanese Society for Cancer of the Colon and Rectum (JSCCR) guidelines 2019 for the treatment of colorectal cancer. Int J Clin Oncol. 2020;25(1):1-42. https://doi.org/10.1007/s10147-019-01485-Z

12. Zhang H, Kang Z, Gong H, Xu D, Wang J, Li Z, Cui X, Xiao J, Meng T, Zhou W, Liu J, Xu H. The digestive system is a potential route of 2019-nCov infection: a bioinformatics analysis based on single-cell transcriptomes. bioRxiv. 2020 [Epub ahead of print]. https://doi.org/10.1101/2020.01.30.927806

13. Guan WJ, Ni ZY, Hu Y, Liang WH, Ou CQ, He JX, Liu L, Shan H, Lei CL, Hui DSC, Du B, Li LJ, Zeng G, Yuen KY, Chen RC, Tang CL, Wang T, Chen PY, Xiang J, Li SY, Wang JL, Liang ZJ, Peng YX, Wei L, Liu Y, Hu YH, Peng P, Wang JM, Liu JY, Chen Z, Li G, Zheng ZJ, Qiu SQ, Luo J, Ye CJ, Zhu SY, Zhong NS; China Medical Treatment Expert Group for Covid-19. Clinical Characteristics of Coronavirus Disease 2019 in China. N Engl J Med. 2020;382(18):1708-1720. https://doi.org/10.1056/NEJMoa2002032

14. Shi Q, Wang P, Hu H, et al. Diagnosis and treatment of emergency digestive endoscopy in Zhongshan hospital of Fudan university during the outbreak of COVID-19. Chin J Dig Endosc 2020. [Epub ahead of print].

15. Young DO, Kumar AS. Local Excision of Rectal Cancer. Surg Clin North Am. 2017;97(3):573-585. https://doi.org/10.1016/j.suc.2017.01.007.

16. Romesser PB, Wu AJ, Cercek A, Smith J, Weiser M, Saltz L, Garcia-Aguilar J, Crane CH. Management of Locally Advanced Rectal Cancer During The COVID-19 Pandemic: A Necessary Paradigm Change at Memorial Sloan Kettering Cancer Center. Adv Radiat Oncol. 2020 Apr 22. https://doi.org/10.1016/j.adro.2020.04.011.

17. van der Valk MJM, Hilling DE, Bastiaannet E, MeershoekKlein Kranenbarg E, Beets GL, Figueiredo NL, Habr-Gama A, Perez RO, Renehan AG, van de Velde CJH; IWWD Consortium. Long-term outcomes of clinical complete responders after neoadjuvant treatment for rectal cancer in the International Watch \& Wait Database (IWWD): an international multicentre registry study. Lancet. 2018;391(10139):2537-2545. https://doi.org/10.1016/S0140-6736(18)31078-X

18. Habr-Gama A, Sabbaga J, Gama-Rodrigues J, São Julião GP, Proscurshim I, Bailão Aguilar P, Nadalin W, Perez RO. 
Watch and wait approach following extended neoadjuvant chemoradiation for distal rectal cancer: are we getting closer to anal cancer management? Dis Colon Rectum. 2013;56(10):1109-17. https://doi.org/10.1097/DCR.0b013e3182a25c4e

19. Dehal A, Graff-Baker AN, Vuong B, Fischer T, Klempner SJ, Chang SC, Grunkemeier GL, Bilchik AJ, Goldfarb M. Neoadjuvant Chemotherapy Improves Survival in Patients with Clinical T4b Colon Cancer. J Gastrointest Surg. 2018;22(2):242-249. https://doi.org/10.1007/s11605-017-3566-z

20. Chang W, Feng Q, Zhu D, et al. Whole-course management strategy for colorectal cancer patients during COVID-19 epidemic. Chin J Digest Surg. 2020;19:E004.

21. Prachand VN, Milner R, Angelos P, Posner MC, Fung JJ, Agrawal N, Jeevanandam V, Matthews JB. Medically Necessary, Time-Sensitive Procedures: Scoring System to Ethically and Efficiently Manage Resource Scarcity and Provider Risk During the COVID-19 Pandemic. J Am Coll Surg. 2020:S1072-7515(20)30317-3. https://doi.org/10.1016/j.jamcollsurg.2020.04.011

22. Coccolini F, Tartaglia D, Puglisi A, Giordano C, Pistello M, Lodato M, Chiarugi M. SARS-CoV-2 is present in peritoneal fluid in COVID-19 patients. Ann Surg. 2020 [Epub ahead of print].

23. Wang W, Xu Y, Gao R, Lu R, Han K, Wu G, Tan W. Detection of SARS-CoV-2 in Different Types of Clinical Specimens. JAMA. 2020;323(18):1843-4.

24. National Center for Immunization and Respiratory Diseases (NCIRD), Division of Viral Disease. Guidance for U.S. Healthcare Facilities about Coronavirus (COVID-19) [Internet]. 2020 [acceso el 3 de abril de 2020]. Disponible en: https://www.cdc.gov/coronavirus/2019ncov/hcp/us-healthcare-facilities.html

25. American College of Surgeons. Clinical Issues and Guidance [Internet]. ACS. 2020 [acceso el 3 de abril de 2020]. Disponible en: https://www.facs.org/covid-19/clinicalguidance

26. Asociación Colombiana de Infectología. Instituto de Evaluación Tecnológica en Salud. Consenso colombiano de atención, diagnóstico y manejo de la infección por SARSCOV-2/COVID-19 en establecimientos de atención de la salud. Infectio. 2020;24(3):1-153.

27. Wexner SD, Cortés-Guiral D, Gilshtein H, Kent I, Reymond MA. COVID-19: impact on colorectal surgery. Colorectal Dis. 2020;22(6):635-640. https://doi.org/10.1111/codi.15112

28. Chinese Society of Colorectal Surgery, Chinese Society of Surgery, Chinese Medical Association, Colorectal and Anal Surgery Committee, Chinese Research Hospitals Association. Chinese expert consensus on surgical diagnosis and treatment strategies for colorectal cancer patients during novel coronavirus pneumonia epidemic. Chin J Pract Surg. 2020;40:241-4.

29. Mintz Y, Arezzo A, Boni L, Chand M, Brodie R, Fingerhut $A$; and the Technology Committee of the European
Association for Endoscopic Surgery. A Low Cost, Safe and Effective Method for Smoke Evacuation in Laparoscopic Surgery for Suspected Coronavirus Patients. Ann Surg. 2020 Apr 13:10.1097/SLA.0000000000003965. https://doi.org/10.1097/SLA.0000000000003965

30. Zheng $\mathrm{MH}$, Boni L, Fingerhut A. Minimally Invasive Surgery and the Novel Coronavirus Outbreak: Lessons Learned in China and Italy. Ann Surg. 2020:10.1097/ SLA.0000000000003924. https://doi.org/10.1097/SLA.0000000000003924

31. Tomita Y, Mihashi S, Nagata K, Ueda S, Fujiki M, Hirano M, Hirohata T. Mutagenicity of smoke condensates induced by CO2-laser irradiation and electrocauterization. Mutat Res. 1981;89(2):145-9.

32. Hensman C, Baty D, Willis RG, Cuschieri A. Chemical composition of smoke produced by high-frequency electrosurgery in a closed gaseous environment. An in vitro study. Surg Endosc. 1998;12(8):1017-9. https://doi.org/10.1007/s004649900771

33. Gloster HM Jr, Roenigk RK. Risk of acquiring human papillomavirus from the plume produced by the carbon dioxide laser in the treatment of warts. J Am Acad Dermatol. 1995;32(3):436-41. https://doi.org/10.1016/0190-9622(95)90065-9

34. Society of American Gastrointestinal and Endoscopic Surgeons SAGES. SAGES and EAES Recommendations Regarding Surgical Response to COVID-19 Crisis [Internet]. 2020 [citado 3 abril 2020]. Disponible en: https://www.sages.org/recommendationssurgical-response-covid-19/

35. Gustafsson UO, Scott MJ, Hubner M, Nygren J, Demartines N, Francis N, Rockall TA, Young-Fadok TM, Hill AG, Soop M, de Boer HD, Urman RD, Chang GJ, Fichera A, Kessler H, Grass F, Whang EE, Fawcett WJ, Carli F, Lobo DN, Rollins KE, Balfour A, Baldini G, Riedel B, Ljungqvist O. Guidelines for Perioperative Care in Elective Colorectal Surgery: Enhanced Recovery After Surgery $\left(\right.$ ERAS $\left.^{\circledR}\right)$ Society Recommendations: 2018. World J Surg. 2019;43(3):659-695. https://doi.org/10.1007/s00268-018-4844-y

36. Contreras CM, Metzger GA, Beane JD, Dedhia PH, Ejaz A, Pawlik TM. Telemedicine: Patient-Provider Clinical Engagement During the COVID-19 Pandemic and Beyond. J Gastrointest Surg. 2020:1-6. https://doi.org/10.1007/s11605-020-04623-5

37. Pinilla R, Caycedo A, Castro JM, Fuentes MA. Manejo del cáncer colorrectal durante la pandemia por SARS-COV2. Rev Colomb Cir. 2020;35(2):235-43. https://doi.org/10.30944/20117582.629

38. Elementos de protección personal que debemos usar en la atención de pacientes durante la pandemia COVID -19 [internet]. Asociación Colombiana de Infectología. 2020 [acceso el 3 de abril de 2020]. Disponible en: https://www.acin.org/index.php/guias/ coronavirus 